\title{
Caracterización Eléctrica de Membranas Poliméricas PVDF/ $\mathrm{CF}_{3} \mathrm{COOLi}$
}

\section{Electrical characterization of polymeric membranes $\mathrm{PVDF} / \mathrm{CF}_{3} \mathrm{COOLi}$}

\section{Caracterização Elétrica de Membranas Poliméricas $\mathrm{FP} / \mathrm{CF}_{3} \mathrm{COOLi}$}

\author{
Rodrigo Andrés Vásquez-Bonilla ${ }^{1}$, Nori Magali Jurado-Meneses ${ }^{2}$, Miguel Iban Delgado-Rosero ${ }^{3}$
}

Forma de citar: R. A. Vásquez, N. M. Jurado, M. I. Delgado, "Caracterización Eléctrica de Membranas Poliméricas PVDF/CF ${ }_{3}$ COOLi”, Respuestas, vol. 20, no. 1, pp. 125 - 130, 2015.

Recibido:

26 de Agosto 2014

Aceptado:

13 de Noviembre 2014

'Magister en Ciencia-Física ravasquezb@ut.edu.co Universidad del Tolima Ibagué-Colombia

${ }^{2}$ Magister en Ciencia-Física Universidad del Tolima Ibagué-Colombia

${ }^{3}$ Doctor en Física Universidad del Tolima Ibagué-Colombia

\section{Resumen}

Objetivo: Este documento estudia los efectos del trifluoroacetato de litio $\left(\mathrm{CF}_{3}\right.$ COOLi) al usarse en una matriz de Polifluoruro de vinilideno (PVDF) para mejorar la conductividad iónica para usarse como electrolito para dispositivos electroquímicos. Metodología: Para esto se prepararon membranas en diferentes combinaciones por el método de solución. Las muestras se caracterizaron por espectroscopía de impedancias entre temperaturas de $298 \mathrm{~K}$ y $373 \mathrm{~K}$ con el fin de identificar el comportamiento de la conductividad de acuerdo a la concentración. Resultados: Los gráficos de conductividad de en función del inverso de la temperatura, muestran un comportamiento Vogel-Tammann-Fulcher (VTF) con pseudo-energías de activación entre $7.30 \times 10^{-3}$ y $5.05 \times 10^{-4} \mathrm{eV}$. Conclusiones: La máxima conductividad obtenida fue de $3.85 \times 10^{-4} \mathrm{~S} \mathrm{~cm}^{-1}$ a temperatura ambiente para altas concentraciones de $\mathrm{CF}_{3}$ COOLi, siendo este valor comparable con resultados obtenidos en otros estudios.

Palabras clave: Electrolito sólido, Membrana de compuesto $\mathrm{CF}_{3} \mathrm{COOLi} / \mathrm{PVDF}$, Polímero iónicos electro-activos.

\begin{abstract}
Objective: This paper studies the effects of lithium trifluoroacetate $\left(\mathrm{CF}_{3} \mathrm{COOLi}\right)$ when used in a Polyvinylidene fluoride (PVDF) membrane to improve its ionic conductivity for use as electrolyte for electrochemical devices. Method: $\mathrm{CF}_{3} \mathrm{COOLi} / \mathrm{PVDF}$ Ionic conductive membranes have been prepared by solution method. The samples were characterized by impedance spectroscopy (IS) with temperatures ranging from 298 $\mathrm{K}$ at $373 \mathrm{~K}$. Results: The plots of conductivity with the inverse of temperature show an Vogel-Tamman-Fulcher (VTF) behavior, with pseudo activation energies between $7.30 \times 10^{-3}$ y $5.05 \times 10^{-4} \mathrm{eV}$. Conclusions: The higther conductivity $\left(3.848 \times 10^{-4} \mathrm{~S}\right.$ $\mathrm{cm}^{-1}$ ) was obtain at room temperature to higth concentration of $\mathrm{CF}_{3} \mathrm{COOLi}$, this values is similarity with results from other documents.
\end{abstract}

Keywords: Solid electrolytes, $\mathrm{CF}_{3} \mathrm{COOLi} / \mathrm{PVDF}$ composite membranes, Ionic electroactive polymers. 


\section{Resumo}

Objetivo: Este documento estuda os efeitos do trifluoroacetato de lítio $\left(\mathrm{CF}_{3} \mathrm{COOLi}\right)$ usado numa matriz de Fluoreto de polivinilideno (FP) para melhorar a condutividade iónica e utilizar-se como eletrólito para dispositivos eletroquímicos. Metodologia: Para isto se prepararam membranas em diferentes combinações pelo método de solução. As amostras se caracterizaram por espectroscopia de impedâncias entre temperaturas de $298 \mathrm{~K}$ e 373 $\mathrm{K}$ com o fim de identificar o comportamento da condutividade de acordo à concentração. Resultados: As figuras de condutividade de em função do inverso da temperatura, mostram um comportamento Vogel-Tammann-Fulcher (VTF) com pseudo-energias de ativação entre $7.30 \times 10-3$ e $5.05 \times 10-4$ eV. Conclusões: A máxima condutividade obtida foi de $3.85 \times 10-4 \mathrm{~S} \mathrm{~cm}-1$ a temperatura ambiente para altas concentrações de $\mathrm{CF}_{3} \mathrm{COOLi}$, sendo este valor comparável com resultados obtidos em outros estudos.

Palavras-chave: Eletrólito sólido, Membrana de composto $\mathrm{CF}_{3} \mathrm{COOLi} / \mathrm{FP}$, Polímero iónicos eletroativos.

\section{Introducción}

Existe mucho interés en la preparación y estudio de electrolitos sólidos poliméricos con altos valores de conductividad, buenas propiedades mecánicas y estabilidad térmica, dado las múltiples aplicaciones en dispositivos electroquímicos [1], [6].

Las soluciones sólidas formadas por polímeros y sales han mostrado adecuadas propiedades para aplicaciones en baterías recargables, sensores de humedad y ventanas electrocrómicas [6]. Con el propósito de mejorar estas propiedades, se han utilizado polímeros con diferentes funcionalidades tales como poli-óxido de etileno (PEO) [8], alcohol polivinílico (PVAL) [5], polifluoruro de vinilideno (PVDF) [11] entre otros.

El PVDF es un polímero termoplástico químicamente inerte, con alta resistencia a la exposición de ácidos, bases y disolventes. Con la ayuda de plastificantes poliméricos como el carbonato de etileno (CE), carbonato de propileno (CP) y diferentes sales $\left(\mathrm{LiBF}_{4}\right.$ y $\left.\mathrm{LiClO}_{4}\right)$ se han obtenido electrolitos poliméricos con valores de conductividad de $3.4 \times 10^{-4} \mathrm{~S} \mathrm{~cm}^{-1}$ para $\mathrm{LiBF}_{4}$ y $15.6 \times 10^{-3} \mathrm{~S} \mathrm{~cm}^{-1}$ para $\mathrm{LiClO}_{4}$ con adecuadas propiedades mecánicas para usarse en celdas combustible [7], [10], [14]. Este polímero es buen disociador de sales de litio lo que permite generar una gran cantidad de portadores de carga para la conducción [9], haciendo de este un excelente candidato para la preparación de membranas conductoras.

Diferentes sales de litio, sodio y potasio se han combinado con polímeros buscando incrementar los valores de conductividad y obtener nuevos sistemas con mejores propiedades mecánicas térmicas $\mathrm{y}$ electroquímicas. Las sales de litio más usadas han sido $\mathrm{LiClO}_{4}$ y $\mathrm{LiCF}_{3} \mathrm{SO}_{3}$ las que se han combinado con PEO y otros polímeros que contienen oxígeno obteniéndose altos valores de conductividad.

Sin embargo, algunas sales orgánicas como los trifluoroacetatos $\left(\mathrm{CF}_{3} \mathrm{COONa} \mathrm{y}\right.$ $\mathrm{CF}_{3} \mathrm{COOLi}$ ) han presentado también valores de conductividad comparable a las anteriores con formación de nuevos complejos poliméricos [11], [12]. Por su pequeña solubilidad el $\mathrm{CF}_{3} \mathrm{COOLi}$ ha sido foco de varias investigaciones $\mathrm{y}$ publicaciones en el estudio de baterías de ion litio mostrando estabilidad de la conductividad en condiciones de almacenamiento controladas [13]. 
Como un nuevo sistema se ha sintetizado y caracterizado eléctricamente con conductividades comparables a los sistemas ya mencionados, membranas de polifluoruro de vinilideno combinadas con $\mathrm{CF}_{3} \mathrm{COOLi}$. $\mathrm{La}$ sintetización se llevó a cabo mediante el método de solución y se obtuvieron membranas con espesores desde $90 \mu \mathrm{m}$, pudiéndose observar (a simple vista) buena resistencia a la tracción y compresión. La caracterización eléctrica evidencio altos valores de conductividad $\left(3.85 \times 10^{-4} \mathrm{~S} \mathrm{~cm}^{-1}\right)$ para altas concentraciones de $\mathrm{CF}_{3} \mathrm{COOLi}$ lo que podría usarse como separadores electrolíticos.

\section{Materiales y métodos}

\subsection{Preparación de muestras}

Las membranas se prepararon por el método de solución por ser una metodología sencilla y de bajo costo que no requiere de equipos sofisticados. Se utilizó PVDF de la casa ALDRICH (99.9\%) en polvo y Tetrahidrofurano (THF) como disolvente. $\mathrm{Se}$ combinó con $\mathrm{CF}_{3} \mathrm{COOLi}$ granulado $(95 \%)$ de la misma casa. Se disolvió la sal $\mathrm{CF}_{3} \mathrm{COOLi}$ en THF por agitación magnética a temperatura ambiente por 30 minutos. Para evitar la evaporación durante la agitación, se usó un balón con retorno del solvente logrando incrementar la temperatura de la solución hasta $323 \mathrm{~K}$ agitándose durante 4 horas. Las dos soluciones se mezclan en recipiente común y se continúa su agitación durante 24 horas.

La solución obtenida se depositó en caja de Petri y se ubicó en un desecador con silica gel para evaporación lenta del solvente. Después de 24 horas se obtuvieron membranas flexibles semitransparentes entre 90 y $120 \mu \mathrm{m}$ de espesor.

\subsection{Medidas de Impedancia}

De las membranas obtenidas se recortaron discos de $5 \mathrm{~mm}$ de diámetro y se ubicaron entre electrodos de platino de esta misma dimensión. Para tomar las medidas de impedancia se utilizó una celda en acero calentada por un bombillo halógeno de 35 watts alimentado por una corriente alterna de $110 \mathrm{v}$.

Las medidas de impedancia se realizaron utilizando un impedanciómetro HIOKI 353250 en un rango de frecuencias de $42 \mathrm{~Hz}$ a 5 $\mathrm{MHz}$ variando la temperatura desde $298 \mathrm{~K}$ hasta $373 \mathrm{~K}$ en intervalos de $10 \mathrm{~K}$. Se controló la humedad relativa del ambiente al 30\% utilizando una trampa de silica por la cual se circulaba el aire contenido en un recipiente hermético en el que se encontraba la celda.

\section{Resultados y análisis}

En las Figura 1 y Figura 2 se muestra los diagramas de Nyquist de membranas de polímero puro y combinado en distintas concentraciones de sal respectivamente. Se puede observar en la Figura 1 que el PVDF puro presenta comportamiento capacitivo, presentado un cambio en su comportamiento dieléctrico a temperatura cercana al punto de fusión de la fase cristalina ( $438 \mathrm{~K}$ ); este cambio es ocasionado por el cambio de fase presente en la membrana a esta temperatura.

En la Figura 2 las medidas realizadas a temperatura ambiente muestran un notable decrecimiento en la resistencia representada por la intersección del semicírculo con la línea horizontal. Este efecto es ocasionado por el incremento en el número de portadores de carga cuando se incrementa el contenido de sal.

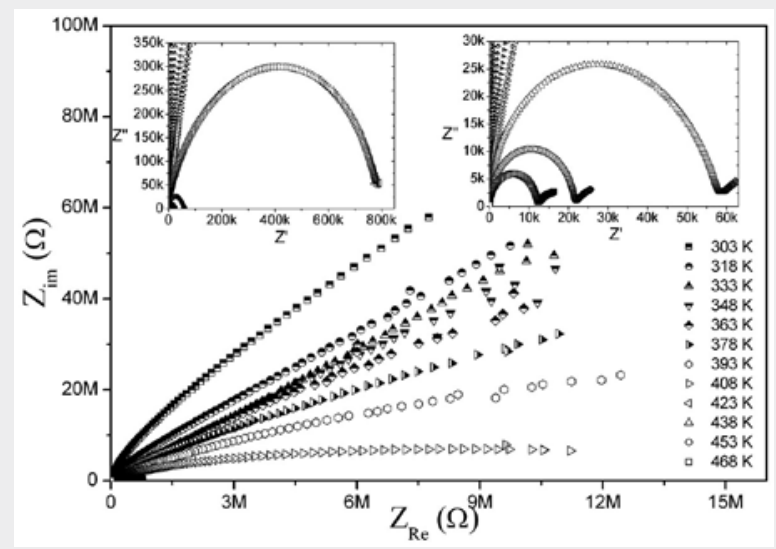

Figura 1. Diagrama de NYQUIST de PVDF a distintas temperaturas Fuente: Autor 
Vol. 20

No. 1

Enero - Junio 2015 ISSN 0122-820X

PP: $125-130$
La Figura 3 muestra los diagramas de Bode para la conductividad real a diferentes temperaturas para una razón de masa polímero/sal de 4:1. En estos gráficos puede observarse una región en la que la conductividad real permanece constante para un rango de frecuencia de $2 \times 10^{3} \mathrm{~Hz}$ hasta $5 \times 10^{5} \mathrm{~Hz}$. A mayores valores de frecuencia puede observarse un crecimiento potencial característico de estos compuestos [2], [4], [8], el cual se ajusta con la relación de Joncher, Ecuación (1), para obtener los valores de conductividad dc $\left(\boldsymbol{\sigma}_{\mathrm{dc}}\right)$.

$$
\sigma^{\prime}=\sigma_{\mathrm{dc}}\left[1+\left(\frac{\omega}{\omega_{\mathrm{p}}}\right)^{\mathrm{n}}\right]
$$

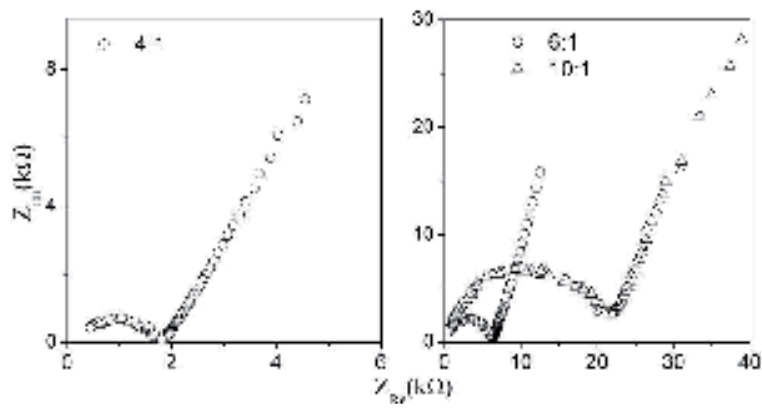

Figura 2. Diagrama de NYQUIST para distintas concentraciones de $\mathrm{PV} \mathrm{DF} / \mathrm{CF}_{3} \mathrm{COOLi}$ a temperatura ambiente

Fuente: Autor

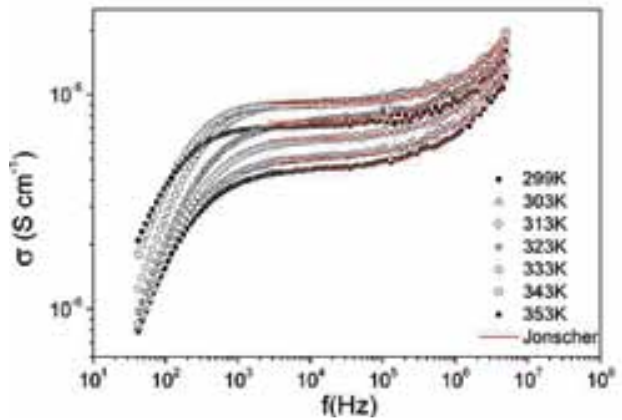

Figura 3. Ajuste JONSCHER para PV DF/ $\mathrm{CF}_{3} \mathrm{COOLi}$ en concentración 4:1

Fuente: Autor

La Figura 4 muestra la conductividad $\sigma_{\mathrm{dc}}$ en función del inverso de la temperatura para las diferentes concentraciones analizadas. Se puede observar un comportamiento VTF, característico de sistemas con alto contenido de fase amorfa [2], [3]

$$
\sigma_{d c}=\sigma_{0} \exp \left(-\frac{B}{k\left(T-T_{0}\right)}\right)
$$

Donde $\sigma_{0}$ es el factor pre-exponencial que se relaciona con el número de portadores, $B$ es la energía de pseudo-activación necesaria para la redistribución del volumen libre y $T_{0}$ se conoce como la temperatura de la movilidad cero.

Tabla I. Parámetros de ajuste VTF para combinaciones de

\begin{tabular}{|c|c|c|c|}
\hline Combinación & $4: 1$ & $6: 1$ & 10:1 \\
\hline$B \quad(\mathrm{eV})$ & $7.302 \times 10^{-3}$ & $5.052 \times 10^{-4}$ & $1.096 \times 10^{-3}$ \\
\hline$\sigma_{0}\left(\mathrm{~S} \mathrm{~cm}^{-1}\right)$ & $3.85 \times 10^{-4}$ & $1.03 \times 10^{-5}$ & $5.01 \times 10^{-6}$ \\
\hline $\begin{array}{ll}T_{0} & (\mathrm{~K})\end{array}$ & 243 & 283 & 280 \\
\hline
\end{tabular}
$\mathrm{PVDF} / \mathrm{CF}_{3} \mathrm{COOli}$

Fuente: Autor

La Tabla I muestra los parámetros del ajuste VTF, Ecuación (2), a los resultados experimentales. Se puede observar que el valor de $\mathrm{B}$ decrece con la concentración de $\mathrm{CF}_{3} \mathrm{COOLi}$ lo que es típico en estos electrolítos. De la misma forma, la conductividad eléctrica aumenta con la concentración de $\mathrm{CF}_{3} \mathrm{COOLi}$ lo que se debe al aumento en los portadores de carga en la matriz del polímero correspondiente a la Figura 4

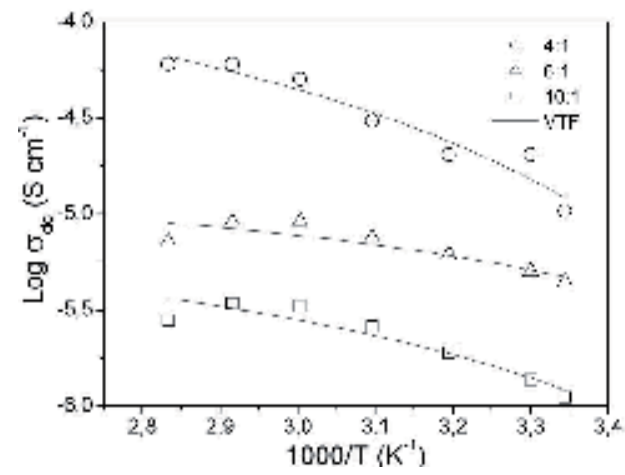

Figura 4. Ajuste VTF para distintas concentraciones de $\mathrm{PVDF} / \mathrm{CF}_{3} \mathrm{COOLi}$

Fuente: Autor

El aumento en la conductividad iónica con la concentración de $\mathrm{CF}_{3} \mathrm{COOLi}$ y el comportamiento capacitivo de la membrana, característico de estos compuestos, es evidencia suficiente para proponerse la combinación $\mathrm{PVDF} / \mathrm{CF}_{3} \mathrm{COOLi}$ para su uso como electrolito para celdas electroquímicas. 


\section{Agradecimientos}

Se expresan los agradecimientos a la oficina de investigación y desarrollo científico de la Universidad del Tolima por todo el apoyo brindado para el desarrollo de la presente investigación.

\section{Conclusiones}

El nuevo sistema polimérico PVDF/ $\mathrm{CF}_{3} \mathrm{COOLi}$ presenta altos valores de conductividad por lo que puede llegar a usarse como membrana electrolítica en dispositivos electroquímicos como baterías y ventanas electrocrómicas.

La combinación de PVDF con $\mathrm{CF}_{3} \mathrm{COOLi}$ presenta un comportamiento Vogel-TammennFulcher (VTF) en un rango de temperaturas entre el ambiente $(303 \mathrm{~K})$ y $357 \mathrm{~K}$. Esto muestra que la conducción iónica que muestra la membrana es un proceso de activación térmica que ocurre desde temperaturas cercanas al ambiente. La temperatura de funcionamiento de la membrana (menor a los $337 \mathrm{~K}$ ) le da la capacidad de ser útil en ambientes de bajas temperaturas.

\section{Referencias}

[1] A. S. Shaplov, D. O. Ponkratov, P-H. Aubert, E. I. Lozinskaya, C. Plesse, A. Maziz, P. S. Vlasov, F. Vidal,Y. S. Vygodskii, "Truly solid state electrochromic devices constructed from polymeric ionic liquids as solid electrolytes and electrodes formulated by vapor phase polymerization of 3,4-ethylenedioxythiophene", Polymer, vol. 55, no. 16, pp. 3385- 3396, 2014.

[2] J. H. Castillo. Fundamentos de caracterización eléctrica de materiales no metálicos mediante circuitos equivalentes, 2010.

[3] M. I. Delgado. "Síntesis y caracterización térmica y eléctrica del complejo polimérico peo/cf3coona". Tesis de Maestría, Universidad del Valle, Departamento de Física, Cali, 2000.

[4] K. P. Padmasree, R. A. Montalvo Lozano, S. M. Montemayor, A. F. Fuentes, "Electrical conduction and dielectric relaxation process in $\mathrm{Ce} 0.8 \mathrm{Y} 0.2 \mathrm{O} 1.9$ electrolyte system", Journal of Alloys and Compounds, vol. 509, pp. 8584-8589, 2011.

[5] B-E. Mellander, M. A. Vargas, R. A. Vargas, "New proton conducting membranes base on $\mathrm{PV} \mathrm{AL} / \mathrm{H}_{3} \mathrm{PO}_{2} /$ H2O", Electrochemical Acta, vol. 44, pp. 4227-4232, 1999.

[6] M. Nádherná, F. Opekar, J. Reiter, K. Štulík, "A planar, solidstate amperometric sensor for nitrogen dioxide, employing an ionic liquid electrolyte contained in a polymeric matrix", Sensors and Actuators B, vol. 161, no. 1, pp. 811817, 2012.

[7] N. Muniyandi, N. Kalaiselvi, P. Periyasamy, R. Thirunakaran, B. Ramesh babu, S. Gopukumar, T. Premkumar, N. G. Renganathan, M. Raghavan, "Optimisation of PVDF-based polymer electrolytes", Journal Power Sources, vol. 96, no.1, pp.14-19, 2001.

[8] R. A. Vargas Nori M. Jurado, I. Delgado, "Conductividad iónica en nuevos compositos $(\mathrm{PEO})_{10}(\mathrm{CF} 3 \mathrm{COONa})-\mathrm{X}$ $\% \mathrm{Al}_{2} \mathrm{O}_{3}$ ", Universitas Scientiarum, vol. 18, no. 2, pp.173-80, 2013.

[9] M. Hema, P. Tamilselvi, "Conductivity studies of $\mathrm{LiCF}_{3} \mathrm{SO}_{3}$ doped PVA: PVdF blend polymer electrolyte", Physica B, vol. 437, pp. 53-57, 2014.

[10] P. Sivakumar, S. Rajendran, "An investigation of $\mathrm{PV} \mathrm{dF} / \mathrm{PV} \mathrm{C}$-based blend electrolytes with EC/PC as plasticizers in lithium battery applications", Physica $B$, vol. 403, no. 4, pp. 509-516, 2008.
Enero - Junio 2015 ISSN 0122-820X PP: $125-130$ 
Vol. 20

No. 1

Enero - Junio 2015 ISSN 0122-820X

PP: $125-130$

[12] M. I. Delgado, N. M, Jurado, R.A. Vargas. "Phase diagram of polymer electrolyte: (x)(PEO)-( 1-x), CF 3 COOLi Diagrama de fases del polímero electrolito: $(\mathrm{x})$ (PEO) - $(1-\mathrm{x}) \mathrm{CF}_{3}$ COOLi", Revista Facultad de Ingeniería, vol. 62, pp. 7782, 2012.

[13] N. M. Jurado, I. Delgado, R. A. Vargas, "Conductividad iónica en nuevos compositos (PEO) ${ }_{10}\left(\mathrm{CF}_{3} \mathrm{COONa}\right)-\mathrm{X} \%$ $\mathrm{Al}_{2} \mathrm{O}_{3}$ ", Universitas Scientiarum, vol. 18, no. 2, pp. 173-180, 2013.

[14] W. A. Van Schalkwijk, B. Scrosati. "Teperature effects on Li-ion cell performance", en Avances in LithiumIon Batteries. New York: Plenum Publishers, 2002, pp. 309-344. 